\title{
MODELLING THE SWITCHING LINK AND MATHEMATICAL OF ACTUAL TRAFFIC
}

\author{
Abed Saif Alghawli \\ Department of Computer Science, Salman Bin Abdulaziz University, Alaflaj, Saudi Arabia
}

Received 2014-03-09; Revised 2014-06-19; Accepted 2014-11-11

\begin{abstract}
The paper represents the research results of studying the switches. The model of a switching link has been suggested. The switch has been presented in the form of a Queuing System (QS), the study of dynamic characteristics of switches has been undertaken and the adequacy of the proposed model of a switching link to actual switches has been valued. The maximum error of the modelling is no more than 5\%, which indicates the sufficient adequacy of the switching link as related to the evaluation of its probability-time characteristics. The accuracy given is eligible for practical and engineering research works. The self-similar traffic characteristics causing an overload rise when passing through the switching links have been studied by means of simulation modelling. The simulation test conducted of the traffic passing through the switching link showed three characteristics to be significant: Intensity, Fano parameter and Hurst exponent.
\end{abstract}

Keywords: Self-Similar Traffic, Switching Link, A Queuing System

\section{INTRODUCTION}

Multiservice networks can often generate powerful spikes. Such localized in time emissions cause substantial packet losses even since the total requirement of all streams is far from the maximum permitted values. Numerous studies results testified that if the input traffic is self-similar, then, whatever self-similar streams multiplexing, the duration of the delays will be high and so larger buffers will be required. Moreover, the larger the values of Hurst exponents are, the higher the requirements for larger buffers will be, even with a slight load factor. If it is necessary to achieve high degree of use, then for self-similar traffic the buffers of larger sizes will be required, even than it has been predicted by the classical queuing analysis (Kolaczyk, 2009; Cai et al., 2007; Song et al., 2005).

This article represents the study of dynamic characteristics of switches, the switch modelling and evaluation of its adequacy to actual switches, as well as the study of the dependence of the queue length in the buffer link on the parameters of the input traffic. The values of the traffic emissions can be considered as the capacity of the buffer memory needed in order to avoid data losses. The cells delayed in the memory can be transferred at the moment of the reduction of the traffic load. Consequently, to achieve the maximum data transmission rate, it is important to determine the optimal buffer size (if the buffer is too small-much of the data will be lost and if the buffer is too large-the recourse gluttony will be caused and the disagreement of network operating parameters will occur).

\section{THE MODEL OF THE SWITCHING LINK}

The actual switch can be provided in different models depending on the degree and completeness of the process specifications and the final objective of the research. The basic functional model of the traffic 
switching node can be created on the basis of the states of the queuing theory. Herewith the switch is in the first approximation is a Queuing System (QS) with the finite or unlimited input buffer and First Input/First Output (FIFO) call servicing discipline. The functional model of the system is shown in Fig. 1.

Consider the model of a high-speed switch based on the Gigabit Ethernet technology, which is the most used in the world (Radaei and Zukarnain, 2009; Guesmi et al., 2006).

Gigabit Ethernet technology transmits the variable length packets at variable packet interval, so the forwarding process of the packet $\mathrm{k}$ will consist of two phases: "p-"-interframe pause and "p +"-packet transmission. The duration of the interframe interval will be denoted as $\tau_{p}[k]$ and the duration of the packet transmission is- $\tau_{f}[k]$.

The duration phase " $\mathrm{p}+$ " is associated with $l[k]$ packet size by the ratio Equation 1:

$$
\tau_{f}[k]=\frac{l[k]}{v_{b c}}
$$

where, $v_{b c}$ - is a bit rate of the channel, the permanent hardware parameter.

Gigabit Ethernet packet consists of a preamble (it is designed to synchronize the transmitter and the receiver of the physical layer), the restricted part and a payload. The size of the preamble is recorded in 512 bits and that of the restricted part in 144 bits. The minimum value of "pure" interframe interval is exactly equal to 96 bits of transmission time which is 96 ns for Gigabit Ethernet and its maximum value is not limited. Considering the fact that the preamble at the link layer is not processed, the value of the "real" minimum interframe interval of 608 ns can be obtained.

The most important parameters of the Gigabit Ethernet link layer are shown in Table 1. As the result, the magnitude lis discrete by its nature and is defined on the bit interval $l \in[512,12144]$ with an increment of 8 bits. The component $\tau_{p}$ is continuous and can be defined on the nanosecond interval $\tau_{p} \in[608, \infty)$.

With the accuracy sufficient for engineering calculations the component $\tau_{p}$ can also be presented as discrete with a sampling interval of $1 \mathrm{~ns}$. The discretization error in this case is less than $2.10^{-3}$. Then the process of traffic forwarding in the communication channel will be discrete both in time and on level.

The manufacturers of network equipment state the bandwidth capacity or similar characteristics as to be the main specifications of the device. This value defines the number of packets that the device can process per unit time in accordance with the requirements of the document IEEE 802.3. The bandwidth capacity may be indicated at different operating conditions. For example, for Cisco's devices the bandwidth capacity is specified in the case of software and fast switching.

For every switch the particular midfrequency of sending and receiving packets is assigned, which is $\sim 19.5 \mathrm{kHz}$. The midfrequency can be pre-set as equal to all switches in the network.

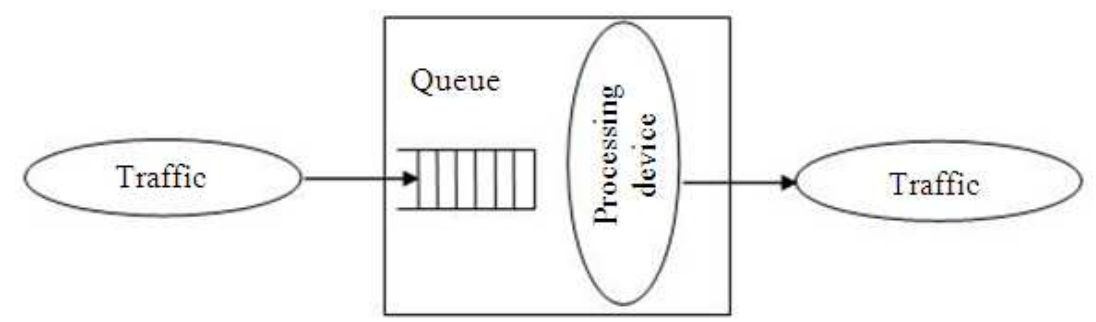

Fig. 1. Functional diagram of the switch in terms of QS

Table 1. Gigabit ethernet link layer parameters

\begin{tabular}{lll}
\hline Parameter & Minimum length packet & Maximum length packet \\
\hline Packet size without preamble & 64 bytes & 1518 bytes \\
Payload & $368 \mathrm{bits}$ & $12000 \mathrm{bits}$ \\
Payload share & $71,88 \%$ & $98,81 \%$ \\
Minimum period of packet flow & $1,120 \mathrm{MSc}$ & $12,752 \mathrm{MSc}$ \\
Maximum frequency of packet flow & $892857 \mathrm{packet} / \mathrm{s}$ & $78419 \mathrm{packet} / \mathrm{s}$ \\
Maximum net bandwidth & $328,571 \mathrm{MB} / \mathrm{s}$ & $941,028 \mathrm{MB} / \mathrm{s}$ \\
\hline
\end{tabular}


Modern switches are multi-tasking and multi-user systems with several network interfaces. Therefore, they can simultaneously transmit and receive dozens of packets (depending on the number of network interfaces in the switch). For this reason, the frequency of sending packets is independent from the pause between packets and their sizes and it can be presented as a determinate value with the accuracy sufficient for the numerical calculations.

Therefore, the switch can be represented as QS, which, in each operating cycle of the system, accepts the input packets received during this working period of the system, processes them and forwards. The model concerned will be denoted as $\mathrm{Y} / \mathrm{D} / \mathrm{C} / \mathrm{h} / \mathrm{d}$, where $\mathrm{Y}$-incoming traffic, Ddeterministic service time equal to one system operating cycle t; C-the system capacity; h-the buffer sizeand d- the discipline applied in the system (Cai et al., 2007).

The finite size of the buffer $h$ means that the buffer can store no more than $h$ packets at any given time $t$. In every window $t$ (window-is the time interval $[t, t+1]$ ) the channel can transmit no more than $\mathrm{C}$ packets which are taken from either buffer or from new incoming packets $\mathrm{Y}_{\mathrm{t}}$. The packet from the buffer which is transmitted in the window $t$ leaves the channel and the whole system concerned at the time $t+1$.

At any time $t$ it is assumed that the events occur in the following order: \{the end of the service in the window $\mathrm{t}-1\} \rightarrow\{$ arrival of new packets ( 0 packets may arrive) $\} \rightarrow\{$ selecting the next packets for the service $\} \rightarrow\{$ packets loss, if it is required by the service discipline $\} \rightarrow$ \{buffering the unlost packets $\} \rightarrow$ opening the window $\mathrm{t}\} \rightarrow\{$ inception of service in the window $\mathrm{t}\}$.

It is assumed that in the model at each time $t$ the discipline decides which of the following alternatives must be applied to each packet in the system:

- Start transmission (service) of the packet at the time $\mathrm{t}$

- Store the packet in the buffer up to when $t+1$

- Reset (lose) the packet at the time $\mathrm{t}$

The most important class of disciplines $d$ is the class satisfying the following conditions:

- If $\mathrm{Y}_{\mathrm{t}}+\mathrm{Z}_{\mathrm{t}}>0$, where $\mathrm{Y}_{\mathrm{t}^{-}}$the number of new packets received at the time $t ; Z_{t^{-}}$the number of packets available in the system at the time $t$ (prior to admission of new packets), then min $\left\{\mathrm{Y}_{\mathrm{t}}+\mathrm{Z}_{\mathrm{t}}, \mathrm{C}\right\}$ packets are to the transmission (service) at the time $\mathrm{t}$

- If $\mathrm{Y}_{\mathrm{t}}+\mathrm{Z}_{\mathrm{t}} \leq \mathrm{h}+\mathrm{C}$, then none of the packets are lost at the time $\mathrm{t}$
- If $\mathrm{Y}_{\mathrm{t}}+\mathrm{Z}_{\mathrm{t}}>\mathrm{h}+\mathrm{C}$, then $\mathrm{Y}_{\mathrm{t}}+\mathrm{Z}_{\mathrm{t}}-\mathrm{h}-\mathrm{C}$ packets are lost at the moment $t$ (it is depended on the particular discipline which packets are lost and which are transmitted)

The following system parameters will be used to assess the impact of incoming traffics on the system.

The value of packet losses for the entire action period of the system:

$$
P=\sum_{i=1}^{N}\left(T_{i}+B_{i}\right)-\left(C+\left(h-B_{i}\right)\right)
$$

Where:

$T_{i}=$ The value of incoming traffic

$B_{i}=$ The number of packets in the buffer at the imoment of time

$C=$ The server capacity

$h=$ The volume (capacity) of the buffer

- The average number of packets in the buffer: $\bar{B}=\sum_{i=1}^{N} B_{i}$

- The loss factor: $k_{L}=\frac{P}{\sum_{i=1}^{N} T_{i}}$

- Buffering factor: $k_{B}=\frac{\bar{B}}{h}$

Before applying this QS for the comprehensive analysis of dynamic characteristics of switches, it is necessary to assess its adequacy to actual switches.

Such studies have been performed in (Polyakov and Kuznetsov, 2005). The common switch "Cisco 3750G" has been used as the real object of the research. The hardware parameters of this device needed for numerical simulation are shown in Table 2.

Table 3 shows the comparative analysis of the average results of the testing and simulating.

The numerical simulation was carried out under the same hardware model parameters as when the assessment of its adequacy. That allows us to bind the results of the simulation to the switch "Cisco 3750G" and so increase the practical significance of the results.

Table 3 shows that the maximum error of the simulation is less than $5 \%$ and this fact suggests the sufficient model adequacy of the prototype object as to the evaluation of its probability-time characteristics. This accuracy is quite allowable to the practical and engineering studies. 
Table 2. Gigabit ethernet switch parameters

\begin{tabular}{ll}
\hline Parameter & RMS value per one port \\
\hline Switching core performance & $1,638 \mathrm{Gbs}$ \\
CPU performance & 1375461 packets/sec \\
Storage capacity of the incoming buffer & $256 \mathrm{~KB}$ \\
\hline
\end{tabular}

Table 3. Results of testing and simulating

\begin{tabular}{llll}
\hline Parameter & Test & Model & Error (\%) \\
\hline Single packet delay, 64 bytes & $3,42 \mathrm{MSc}$ & $3,54 \mathrm{MSc}$ & 3,5 \\
Single packet delay, 1518 bytes & $10,15 \mathrm{MSc}$ & $9,82 \mathrm{MSc}$ & 3,25 \\
Delay (actual traffic, 200 packets) & $7,12 \mathrm{MSc}$ & $6,93 \mathrm{MSc}$ & 2,66 \\
Jitter (actual traffic, 200 packets) & $1,66 \mathrm{MSc}$ & $1,74 \mathrm{MSc}$ & 4,8 \\
\hline
\end{tabular}

\section{ANALYSIS OF THE QUEUES OCCURRING IN THE BUFFER AND THE NUMERICAL EXPERIMENT RESULTS}

The switch model described above can be used to study the queues occurring in the switch buffer and losses when the simulation of the network switching node operation. The work has been dealing with the simulation modelling of the channel load and buffer queues. The input buffer was fed with various input data: The implementation of network traffic and simulative implementations of statistically self-similar stochastic process. The Hurst exponent for such a process coincides with the Hurst exponent of the input traffic but the queue possesses significantly heavier tails than the traffic realization. The incoming traffic $\mathrm{Y}(\mathrm{t})$ will be treated as a random process with an arbitrary probability distribution law.

The conducted simulation modelling has testified that the queue length in the buffer is determined by three main parameters of the traffic: Traffic intensity, Fano parameter and Hurst exponent. The Fano parameter $F$ is defined as the variance ratio of the events count at the given time step $\mathrm{T}$ to the mathematical expectation of this quantity: $F(T)=\frac{D[N(T)]}{M[N(T)]}$, where $N(T$-the random variable determining the number of events of the test flow at the interval T (Clegg, 2006; Radivilova, 2007a; Radaei and Zukarnain, 2009; Cirianni and Leonardi, 2012). The large values of the Fano parameter correspond to the wide spread of values of the input stream, which, even at low intensity, creates queues. The Hurst exponent characterizes the long-term dependence of the process. In particular, this means that high values of the process are likely to be followed with the same high values, which will give no way for the buffer to be cleared fast. The average system workload varied from 70 to $95 \%$ (Kirichenko, 2007; Karpuhin, 2008; 2007; Radivilova, 2007b).

The modelled system of network traffic processing is a server with a variable performance or data processing rate (the number of packets per unit time that can be handled) and a buffer memory (a buffer) with a specified volume (amount of data that can be stored). The server puts the raw traffic (that has not been processed yet at one certain step (unit time) of its operation) into the buffer memory.

The data entering Wide-Area Network from the LAN provider have been used as input data. Osi.dat, Osi.dst and Osi.04.src implementations represent the time dependence of the OSI model on the size of network second level protocol frames (Ethernet-frames in this case), passing through the local area network to the WAN network.

The Osi.dat, Osi.dst, Osi.04.src and Tcp.dat implementations have been taken as the samples of a real traffic. The simulation modelling of forwarding the traffic with the length $n=4096$ of the samples with the parameters $\bar{X}$ (bit/s), Fano F, Hurst $H$ via the communication channel node for the buffer with an infinite size and the buffer with a size of 256000 bits has been conducted for these implementations. The value of the average queue length in the buffer $\bar{B}$ and the value of the tail heaviness parameter of the queue $\alpha_{B}$ have been estimated for the buffer infinite in size. For the buffer limited in size the average length of the queue $\bar{B}_{L}$ and the amount of data loss $\mathrm{P}$ in percentage terms have been determined. Table 4 describes the parameters above mentioned when a system load at $90 \%$.

Figure 2 shows the results of the numerical simulation modelling for different degrees of loading when the specified implementations of traffic were used as the input data.

The basic characteristics have been determined for actual network traffics and, based on them, the simulation modelling of the traffic forwarding through 
the communication channel node has been conducted both for the buffer infinite in size and the buffer of 256000 bits in size.

The value of the average queue length in the buffer $\overline{\mathrm{B}}_{\mathrm{L}}$ and the value of the tail weight parameter of the queue $\alpha_{B}$ have been estimated for the buffer infinite in size. For the buffer limited in size the average length of the queue $\bar{B}_{L}$ and the amount of data loss $P$ in percentage terms have been determined.
When testing the hypothesis for equality of the average queue lengths for the actual traffic and the $|\mathrm{t}|=$ 0.632 criteria of the simulative implementation, the hypothesis has been adopted with the significance level $\alpha=0.05$. The parameter values of the queue tail heaviness for actual and simulative implementations differ by less than $10 \%$. The value of data losses to real and simulative loading coincides to integer values of percents. This fact suggests the conclusion about the adequacy of the model.

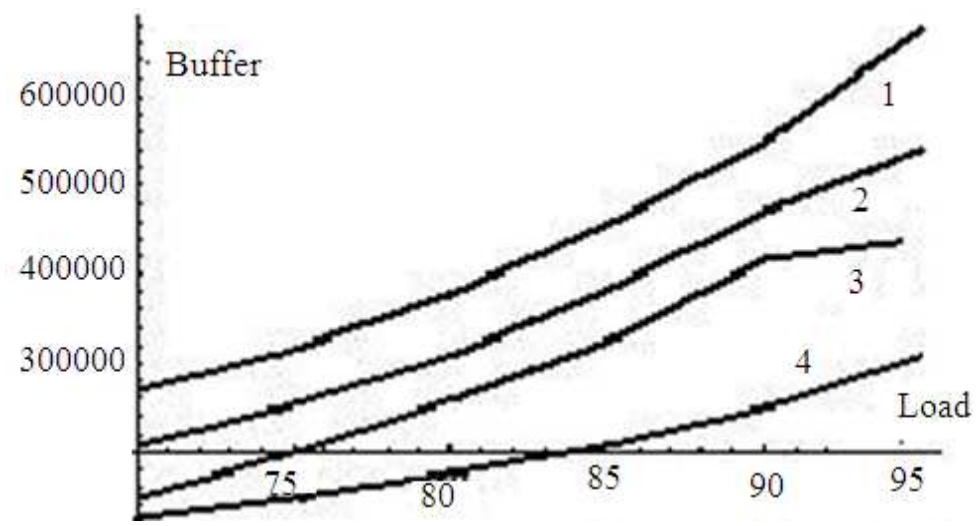

Fig. 2. Dependence of the average queue length on the loading for the actual traffic implementations

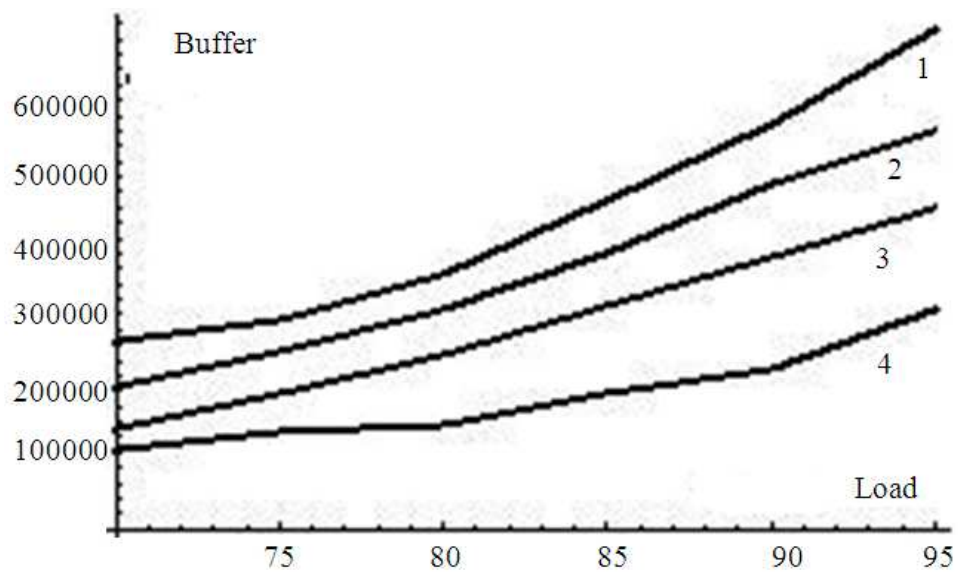

Fig. 3. Dependence of the average queue length on the loading for simulative traffic implementations considering $\bar{X}$, F, $H$ parameters

Table 4. Actual traffic parameters

\begin{tabular}{lllllllll}
\hline Implementation & $\overline{\mathrm{x}}$ & $\mathrm{F}$ & $\mathrm{H}$ & $\overline{\mathrm{B}}$ & $\mathrm{B} / \overline{\mathrm{x}}$ & $\alpha_{\mathrm{z}}$ & $\overline{\mathrm{B}}_{\mathrm{L}}$ & $\mathrm{P}, \%$ \\
\hline Osi.dat (1) & 4500 & 5900 & 0.8 & 540159 & 120 & 0.43 & 73620 & 5.4 \\
Tcp.dat(2) & 4200 & 7200 & 0.86 & 470564 & 112 & 0.27 & 63373 & 7.2 \\
Osi.src (3) & 3700 & 7100 & 0.82 & 410256 & 110 & 0.36 & 75256 & 4.4 \\
Osi.dst (4) & 2700 & 5200 & 0.78 & 251368 & 93 & 0.31 & 81175 & 3.5 \\
\hline
\end{tabular}


Table 5. Parameters of the simulative traffic $y(t)$

\begin{tabular}{lllllllll}
\hline Implementation & $\overline{\mathrm{x}}$ & $\mathrm{F}$ & $\mathrm{H}$ & $\overline{\mathrm{B}}$ & $\mathrm{B} / \overline{\mathrm{x}}$ & $\alpha_{\mathrm{z}}$ & $\overline{\mathrm{B}}_{\mathrm{L}}$ & $\mathrm{P}, \%$ \\
\hline Osi.dat (1) & 4430 & 5850 & 0.8 & 570000 & 126 & 0.41 & 73653 & 5.9 \\
Tcp.dat(2) & 4100 & 7260 & 0.86 & 440000 & 104 & 0.29 & 63378 & 6.7 \\
Osi.src (3) & 3710 & 7130 & 0.82 & 350000 & 95 & 0.35 & 75259 & 4.1 \\
Osi.dst (4) & 2640 & 5280 & 0.78 & 210000 & 78 & 0.33 & 81171 & 3.8 \\
\hline
\end{tabular}

Figure 3 and Table 5 show the results of the numerical modelling for these four simulative implementations.

Each of the implementations being studied had the average value, the Fano parameter and the Hurst exponent coinciding with those for the actual traffic implementations. Obviously, the simulative implementations generate the queues identical to those occurring when actual traffic passing through the channel.

\section{CONCLUSION}

The model of the switching link has been suggested and studied. The switch has been represented as QS, the dynamic characteristics of the switches have been traced, the adequacy of the proposed model of the switching link has been determined by means of the actual switches. The maximum modelling error does not exceed 5\% that proves the sufficient adequacy of the switching link model as to its probability-time characteristics. This accuracy is acceptable for practical and engineering research works.

The characteristics of self-similar traffic, which can cause the overloads when the traffic forwarding through the switched link, have been investigated by means of the simulative modelling. The simulation conducted has shown three characteristics to be significant in the process: intensity, Fano parameter and Hurst exponent.

The scientific novelty of the work testifies that the given model takes into account the dynamic characteristics of the switches, service quality parameters and its adequacy to actual switches; all these factors mentioned allow the applying of the model proposed for the investigation of various characteristics of the network when different data input. The practical relevance of the work consists in that the applying of the model proposed enables the simulation modelling of the network running with any simulated input traffic for the network recourses to be optimized.

\section{REFERENCES}

Cai, H., D. Eun, S. Ha, I. Rhee and L. Xu, 2007. Stochastic ordering for internet congestion control and its applications. Proceedings of the 26th IEEE International Conference on Computer Communications, May 6-12, IEEE Xplore Press, Anchorage, AK., pp: 910-918. DOI: 10.1109/INFCOM.2007.111

Cirianni, F. and G. Leonardi, 2012. Environmental modeling for traffic noise in urban area. Am. J. Environ. $\quad$ Sci., $\quad$ 8: 345-351. DOI: 10.3844/ajessp.2012.345.351

Clegg, R.G., 2006. A practical guide to measuring the Hurst parameter. Int. J. Simulat., 7: 3-14.

Guesmi, H., B. Bouallegue, R. Djemal and R. Tourki, 2006. Design of a high-performance IP switching architecture. J. Comput. Sci., 2: 218-223. DOI: 10.3844 jessp.2006.218.223

Karpuhin, A.V., 2007. Investigation network productivity and the emergence of self-similar load overload when using OPNET Modeler.

Karpuhin, A.V., 2008. Self-similarity in information systems.

Kirichenko, L.O., 2007. Investigation of the influence of self-similarity in the design of traffic network fragment.

Kolaczyk, E.D., 2009. Statistical Analysis of Network Data: Methods and Models. 1st Edn., Springer Science and Business Media, New York, ISBN-10: 0387881468, pp: 398.

Polyakov, S. and N. Kuznetsov, 2005. subscriber access system. Wireless-choice questions: Product catalog, Mode of access to the magazine.

Radaei, T.A.A. and Z.A. Zukarnain, 2009. Comparison study of transmission control protocol and user datagram protocol behavior over multiprotocol label switching networks in case of failures. J. Comput. Sci., 5: 1042-1047. DOI: $10.3844 /$ jcssp.2009.1042.1047

Radivilova, T., 2007a. Modelling of advancing of a selfsimilar network traffic in communication channel. East Eur. J. Adv. Technol., 4: 12-15. 
Radivilova, T.A., 2007b. Modeling self-similar traffic with given characteristics. Proceedings of the 3rd International Young. Scientific-Technical. Conference on Modern Problems of Radio Engineering and Telecommunications, Apr. 17-21, Sevastopol.
Song, C., S. Havlin and H.A. Makse, 2005. Selfsimilarity of complex networks. Nature, 433: 392395. DOI: $10.1038 /$ nature 03248 